\title{
PHASE: a 'health technology' approach to psychological treatment in primary mental health care
}

David Richards, School of Nursing, Midwifery and Health Visiting, University of Manchester, Manchester, UK, Ann Richards, Michael Barkham and Jane Cahill, Psychological Therapies Research Centre, University of Leeds, Leeds, UK and Chris Williams, Department of Psychological Medicine, University of Glasgow, Glasgow, UK

\begin{abstract}
The PHASE research programme is an NHS-Executive funded, randomized controlled trial of assisted self-help for common mental disorders, delivered by practice nurses in primary care. The self-help guide - Managing anxiety and depression: a self-help guide published by the Mental Health Foundation - is conceptualized as a 'health technology' where the nurse's role is to educate patients how to get the best out of the technology, supervise its safe delivery and monitor its continued use by patients so that they can use it independently with little reliance on nurses or other health professionals. Twenty-three nurses were trained on one of five, 3-day courses to deliver PHASE. As the final task during the training course, nurses were asked to describe what they would say to a patient when delivering PHASE. From these data a qualitative thematic content analysis was used to develop a coding framework which showed that nurses were able to articulate a sophisticated health technology rationale, including relevant psychological models and the principle of an evidence base. This study demonstrates that it is possible to equip primary care nurses with both a highly developed understanding and the knowledge to deliver self-help mental health care. Training nurses using this model moves us away from traditional mental health training courses that have attempted to produce 'watered down' versions of specialist practitioners to a more appropriate skills and cultural 'health technology' fit for delivering primary mental health care.
\end{abstract}

Key words: self-help; mental health care; practice nurses; primary care; training

\section{Introduction}

The impact of mental health problems on the economic and social fabric of the UK is huge. Combined prevalence rates for mental health problems mean that at any one time one in six people will be suffering from some form of mental illness ranging from depression to psychosis (Department of Health, 1999). In high income countries such as the UK it is estimated that $23 \%$ of the total burden of disease is due to mental illness (World Health Organization, 1999). The annual cost to England

Address for correspondence: Dr David Richards, School of Nursing, Midwifery and Health Visiting, Coupland III Building, University of Manchester, Oxford Road, Manchester, M13 9PL, UK. Email: David.A.Richards@man.ac.uk alone is thought to be $£ 32$ billion (Patel and Knapp, 1998).

UK health policy has clearly identified mental health as a priority, such that it has formed the subject of one of the first two National Health Service National Service Frameworks (NSF) for England and Wales (Department of Health, 1999). Within the NSF's seven standards for mental health, standards one and two, and to some extent standard three, concentrate on mental health provision from within primary care. Action is proposed to strengthen mental health promotion and primary mental health care for common mental health problems. Although the Mental Health NSF also focuses on serious and enduring mental health problems such as psychosis, the standards that address primary care recognise that $90 \%$ of com- 
mon mental health problems are assessed, treated and cared for in their entirety in primary care (Goldberg and Huxley, 1992). Consultations for the most common mental health problems - anxiety and depression - occupy up to one-quarter of all GP consultations (Goldberg and Bridges, 1987). This rate increases to $40 \%$ of all consultations when sub-syndromal or sub-threshold mental illness is included and the figure is likely to be even higher than this when we consider that frequent attendees for physical problems may have underlying mental health difficulties.

These common mental health disorders are usually managed in primary care without referral to specialist mental health services. Unfortunately, as acknowledged by the Mental Health NSF, effective management of anxiety and depression may be poor due to a lack of interest, skills, knowledge and expertise (Williams et al., 1997). Many attempts have been made to improve the recognition and management of common mental health disorders by general practitioners (GPs). However, the task is enormous given that no more than $40 \%$ of GPs will have experienced a mental health placement during their post-graduate rotational training (Tylee, 1998). Although it has been shown to be possible to train GPs in client-centred, problem-based interviewing for mental health (Gask et al., 1988), the generalizability of such initiatives has been poor. For example, the receipt of a therapeutic dose of antidepressants for patients diagnosed with depression was found to be a minority experience in one study looking at mental health care by GPs in primary care (Donoghue and Tylee, 1996). Other recent studies have also demonstrated only modest or negative findings from implementing clinical practice guidelines for GPs in the management of depression (Thompson et al., 2000; Worrall et al., 1999).

\section{Primary care nurses' involvement in mental health}

Since 1990, the increase in primary care workload has led to a recognition that nurses should play a more diverse and significant role in caring for people within primary care (Richards et al., 2000). One example is the potential for nurses to detect and manage common mental health problems such as depression (Mead et al., 1997). Surveys of prac- tice nurses indicate that around $50 \%$ of patients consulting them for physical health reasons ask for advice about mental health (Gray et al., 1999; Thomas and Corney, 1993). Some mental health interventions delivered by nurses in primary care have been shown to be effective. For example, nurse-delivered drug counselling was almost twice as effective in maintaining antidepressant adherence at 12 weeks compared with GP treatment-asusual and produced significantly better results in patients with major depression prescribed antidepressants (Peveler et al., 1999). Primary care nurses have also been shown to be effective when delivering psychological treatments such as problem solving (Mynors-Wallace et al., 2000).

However, similar problems exist with the training of primary care nurses as for GPs in that previous exposure to mental health in basic training may have been extremely limited or non-existent and both confidence and enthusiasm for such work are often in short supply. Practice nurses consistently report that they fear becoming involved in mental health consultations which may lead to them uncovering problems for which they feel inadequately prepared and ill-equipped to deal with (Gray et al., 1999; Nolan, et al., 1999; Thomas and Corney, 1993).

\section{Self-help for common mental health problems in primary care}

A range of factors has stimulated interest in selfhelp interventions for common mental health problems. These include the sheer volume of need, the reluctance of many patients to take medication (Priest et al., 1996) and the concern of practitioners in secondary care wishing to control their ever increasing waiting lists and respond to NSF calls to focus on severe and enduring mental illness. Behavioural and cognitive psychotherapy researchers, in particular, have demonstrated that the diligence with which patients carry out their own treatment exercises between therapy sessions is an important predictor of treatment success (Marks, 1987). Most commonly, the intervention used consists of bibliotherapy, although some studies have compared other methods of self-help therapy delivery such as computers (Ghosh and Marks, 1987). Data from a recent systematic review (Bower et al., 2001) yielded seven randomized 
controlled trials of self-help interventions for patients with anxiety/stress and depression within primary care, all of which used written material varying in length from two to 79 pages (Donnan et al., 1990; Holdsworth et al., 1996; Kiely and McPherson, 1986; Kupshik and Fisher, 1999; Milne and Covitz, 1988; Sorby et al., 1991; White, 1995). Even though all seven studies showed the same methodological drawbacks commonly seen in evaluations of psychological therapies (such as small sample sizes), the review does suggest that self-help packages perform at least as well as usual care and may even be more effective for some patients compared with GP care. However, the size of the effect, or its advantages in terms of cost, remain to be determined.

The self-care packages used in these studies can be seen as a type of health technology. Health technology is defined as 'any method used by those working in health services to promote health, prevent and treat disease and improve rehabilitation and long-term care. "Technologies" in this context are not confined to new drugs or pieces of sophisticated equipment' (http://www.ncchta.org). Other health technologies are commonplace in primary care where health promotion or self-care are concepts central to primary care nursing. Indeed, delivering efficacious health technologies efficiently is the objective of most health services research.

\section{Two-plus-one model of delivery}

One related strand of research in psychotherapy that has helped focus attention on alternative models of service delivery is the work on the twoplus-one (' $2+1$ ') model. This refers to a system of delivering psychotherapy which aims to give patients a start on the road to recovery - remoralization (i.e., to 'turn the corner') - rather than the expectation of complete cure. It builds on the dose-effect curve which shows the disproportionately high gains made by clients in the early sessions of treatment (Howard et al., 1986). The model comprises two weekly sessions delivered 1 week apart followed by a third session approximately 3 months later and thereby maximizes focused support in the early sessions together with a clear framework for minimizing dependence on the therapist. The model has been evaluated in a randomized control trial where it was found that between 65 and $72 \%$ of patients with sub-clinical and low-level clinical symptoms of depression were improved after delivery of the model (Barkham et al., 1999).

It is unlikely that secondary care mental health nursing services will ever have the resources to manage the vast majority of mental health problems presenting to primary care. Nor is it necessarily desirable, given that there is also little evidence that such specialist mental health services are more effective than routine GP care in the management of common mental health problems (Balestrieri et al., 1988; Friedli and King, 1996; Roth and Fonagy, 1996). In contrast, an approach which combines the potential of practice nurses, self-help approaches and $2+1$ delivery strategies is a possible way of addressing some of the need in primary mental health care identified earlier.

\section{PHASE - psychological health: assessing self-help education in primary care}

The PHASE (psychological health: assessing selfhelp education) primary care research programme is an NHS-Executive funded, randomized controlled trial of supervised self-help for common mental disorders, delivered by practice nurses in primary care (PHASE Group, 1999). It aims to train practice nurses in the delivery of a brief supervised self-help intervention to patients with mild to moderate mental health problems in primary care and to evaluate the new intervention against a control condition comprising treatmentas-usual from the patient's GP. The PHASE programme tests three specific questions: first, the relative efficacy of the self-help intervention package delivered by practice nurses as compared with treatment-as-usual; secondly, whether patients are enabled to manage their own presenting condition effectively and thereby require fewer subsequent primary and secondary consultations; and thirdly, comparing costs between the intervention and treatment-as-usual.

\section{The self-help guide}

The self-help book - Managing anxiety and depression: a self-help guide (Holdsworth and 
Paxton, 1999) - is a 45 page long A5 booklet published by the Mental Health Foundation, an independent mental health research charity. It was developed for people presenting with low to moderate levels of depression or anxiety and tested in a research programme (Holdsworth et al., 1996). It is based on cognitive behavioural principles and the understanding that emotions (principally anxiety and depression) consist of physical, behavioural and cognitive components and as such can be modified by exercises targeting these specific components. The booklet instructs patients how to monitor their emotional health and choose relevant exercises to address their own unique experiences.

Accordingly, the aims of this paper are two-fold: first, to describe the rationale behind the development of a training programme enabling nurses to deliver self-help mental health; and secondly, to present post-training data on practice nurses' ability to explain the self-help programme to patients.

\section{Training: the health technology rationale}

The nurse's aim in PHASE is to assist patients to make the best use of the self-help guide in order to maximize the benefit to patients from the information, instructions and exercises contained within the guide. At all times it is the patient's treatment thus maintaining responsibility for change with the patient. To this end, nurses deliver the book using a $2+1$ session format. During session one, nurses assess the patient using a client-centred, problemfocused interview to determine the individual nature of the patient's difficulties, assess the patient for risk of self-harm, introduce the self-help guide, do an exercise from the guide together and agree homework exercises with the patient. One week later, during session two, nurses review the results of the homework exercises with the client, reassess the patient for risk of self-harm, problem solve any difficulties with the exercises, assist the patient to identify relevant guide exercises specific to their problems and agree homework exercise objectives. Session three, 12 weeks later, is a review of progress and the patient's current health and risk together with a future planning session.
Essentially, like many other health care approaches used in primary care, patients are taught to use the guide themselves. The nurse's role, therefore, is to teach patients how to get the best out of the guide, ensure it is used safely and monitor its continued use by patients so that they can use it independently with little reliance on nurses or other health professionals.

Given the role of the nurse in delivering the guide, a training programme had to be devised which differed radically in concept from most courses educating non-mental health nurses for primary mental health care. Using non-mental health specialists led to a choice between replicating in some way the skills of mental health specialist nurses, albeit in a very dilute form, or educating practice nurses to deliver the guide as a 'health technology'. Nurses are particularly familiar with these concepts in physical care given that they teach patients to manage a range of physical health conditions such as asthma and diabetes using special equipment and patient-administered medication regimes. The health technology approach, therefore, promised to make better use of pre-existing skills and prior knowledge and be a better cultural fit with practice nurses' current practice as opposed to an external paradigm more familiar to secondary mental health practitioners. Given that the self-help guide is designed to be used by people suffering from common mental health problems with minimal or no support from professionals, facilitating its delivery is an obvious candidate for a training programme based on teaching nurses to use it as they would any other piece of 'health technology'.

We therefore devised a training programme which capitalized on the health technology approach. As part of assessing treatment integrity within the PHASE RCT we assessed the practice nurse trainees at the end of the course to judge their understanding of our health technology approach. We did this by evaluating how they would deliver it to patients in order to answer the following research question: 'How will practice nurses explain self-help mental health care to patients after they have attended a training programme based on a "health technology" model of self-help delivery?' 


\section{Method}

\section{Sample}

The sample comprised 23 practice nurses recruited from those practices which had agreed to take part in the main PHASE trial. The average age of nurses was 43.1 years (range 31-59). All nurses were registered in adult nursing with five previously having started their careers as enrolled nurses. Four nurses had a midwifery and two a sick children's qualification, four were district nurse trained and one a health visitor. They had been professional nurses for an average of 19.6 years (range 4-39) and practice nurses for an average of 8.3 years (range 1-26). Although most nurses had additional professional qualifications (for example, in asthma and/or diabetes management and family planning certificates) only three nurses had formal practice nurse qualifications. Four nurses were trained to bachelors' level. In terms of mental health qualifications only one nurse had a registered mental health nursing qualification, a further two had basic counselling certificates and one had recently taken a level three academic module in mental illness with specific reference to primary care. Seventeen nurses worked with other nurses in their surgeries - 12 part time, five full time. Six nurses worked alone - two part time and four full time.

\section{Training programme}

The practice nurses attended one of five 3-day training courses organized between January and June 2000. Each training course required practice nurses to attend for 1 day each week for 3 consecutive weeks. The objectives of the training courses were three-fold: first, for the nurses to become familiar with the self-help guide; secondly, for them to understand the principles upon which it is based; and thirdly, to become competent in teaching patients how to use the guide safely to selftreat their mental health problems. Each training day featured skills practice sessions where nurses rehearsed delivering the guide using the $2+1$ framework. The training cohorts comprised no more than eight nurses, and all skills practice sessions were facilitated by an experienced mental health practitioner (DR, $\mathrm{AR}, \mathrm{MB}$ and $\mathrm{CW}$ ) in groups of three or four nurses.

\section{Task}

As the final task during the training course, all practice nurses were asked to answer an openended question and write down their answers on paper. They did this without conferring with each other or to the notes made during the training. The question was: 'Please imagine you are presenting the self-help manual as delivered in the $2+1$ model to a patient. What would you say to them to enable them to use it?'

\section{Analysis}

The objective of the analysis was to describe the themes apparent in the content of the nurses' answers in order to assess nurses' understanding of how they would deliver the PHASE mental health programme to patients. In choosing an analysis method we wished to avoid a priori assumptions about the nurses' answers and so refrained from applying a predetermined coding framework. Instead, we chose a qualitative method of content analysis in order to develop a coding frame for analysis based on the nurses' own answers rather than one developed by the trainers beforehand.

\section{Coders}

Using the thematic content analysis technique of Burnard (1991), two members of the research team (first and second authors) independently examined the answer texts. Both researchers had been involved in the training, one as a trainer (DR), the other as an administrator and 'patient' for role play skills practice during the training (AR). Both researchers were nurses with specialist mental health clinical training and experience.

\section{Data analytic procedures}

All elements of potential data from the text were extracted and transferred to a separate data sheet (data sheet 1) - excluding only words and phrases which added no additional meaning to the data. The data sheet was scanned carefully and common words or phrases were transferred to a second data sheet (data sheet 2). Statements expressing similar concepts were grouped together. Data sheet 2 was then examined and groups of statements which seemed to refer to very similar ideas put together under general thematic headings which tried to be as separate as they could from each other and reflect quite different areas of thought and opinion. The development of data sheets 1 and 2 was 
achieved through a combination of manifest theme identification (where common words or phrases occurred very frequently) and inference (requiring understanding and interpretation from the researchers). Differences in coding and grouping were resolved iteratively through discussion between the two coders and reference to the source answer texts. This resulted in much swapping and recategorization as overarching explanatory headings developed, based directly on the gradual reduction of the original data. Definitions were then written for both overall explanatory themes and initial concept groupings.

Individual practice nurses' answer data were then examined using the framework of explanatory themes and concept groupings developed above. Counts were made of occurrences of statements which could be classified under each theme and concept. Frequencies were then calculated for each theme and concept.

\section{Results}

We present the results in two sections. The first focuses on the thematic content analysis of the nurses' responses and the second focuses on the frequencies of practice nurses' use of explanatory themes.

\section{Thematic content analysis}

Four major explanatory themes arose from the analysis: 1) the guidebook, 2) the validity of the programme, 3) models underpinning the programme, and 4) the nurses role in the programme.

The results from each of these themes are explicated below:

\section{The guidebook}

This theme refers to instances of descriptions of the guide as a whole, within which nurses described four concepts: 1) purpose, 2) definition, 3 ) structure and 4) ease of use. Nurses described the purpose of the book as to 'help people with common mental health problems' or 'to benefit their particular problem' or 'explain mental health'. They defined the book as 'a self-help manual' to 'use as you like'. The contents and structure of the book were discussed by referring to the way the guide is set out including many of the exercises in the guide, for example, 'useful contents and index section', 'practical exercises', 'relevant selfhelp exercises'. The ease of use of the guide was suggested by comments such as 'simple to use', 'easy to read' and 'written in plain English'.

\section{The validity of the programme}

This theme referred to nurses' articulation of a rationale behind the guide based on a range of sources of evidence and legitimacy. The five concepts in this theme were: 1) the guide's formal and 2) informal evidence bases; 3) the legitimacy of those who had written and 4) published it; and 5) the fact that the guide was part of a research study. Nurses referred to the formal evidence base through statements such as 'evidence-based instructions and exercises' and 'research-based'. Less formal evidence of effect was suggested by comments such as 'people have found it useful' and 'worked well with others'. The fact that the guide was written by mental health specialists was drawn to attention using statements such as 'written by two credited professionals'. Nurses also noted that the guide was 'backed by the Mental Health Foundation', a 'respected body'. Finally, there was some reference to the guide being 'part of a research study' within this theme.

\section{Models underpinning the programme}

Nurses articulated three concepts which can be regarded as examples of theoretical models which underpin the guidebook and the PHASE programme's approach to mental health care. The concepts were: 1) the three systems model of emotion (autonomic, behavioural and cognitive) for example, "explain about feelings, thoughts and behaviour', 'we are able to describe our problems more accurately if we use feelings, thoughts and behaviours as a guide'. 2) The $2+1$ model of service delivery was also referred to specifically, for example 'see [the patient] today, in a week and in three months'. 3) Finally, nurses referred to remoralization using predictions of progress, for example 'may feel up one day, down the next, this is normal' and 'how it may work with ups and downs'.

\section{The nurse's role in the programme}

The final themes concerned the manner in which nurses operate within the PHASE programme with four underlying concepts of the nurse as: 1) guide, 2) explicator, 3) collaborator and 4) reviewer. 
Nurses explained that they would 'help you [the patient] find your way around it [the guidebook]' and that they would be 'here as a guide'. Nurses said they would explain the guide by 'checking the patient understands they know what they are doing' and 'enable greater understanding'. The collaborative role of the nurse was explained by reference to in-session activities such as one of the early exercises 'do tracking exercise' and 'do exercises together'. Finally, the role of the nurse in reviewing progress through discussions about 'homework' exercises was indicated through comments such as 'ensure homework complete' and 'set homework'.

\section{Frequencies of practice nurse use of explanatory themes}

Application of the framework above to individual answer texts produced the following results. Table 1 details the numbers of nurses using each of the four explanatory themes in their suggested explanations to patients. This shows that all nurses used the guidebook theme with the other themes mentioned by more than $80 \%$ of nurses. Table 2 details the numbers of nurses using various combinations of the four explanatory themes and the missing elements from these combinations. This shows that $70 \%$ of nurses used all four themes in their explanations with only three nurses using less than three themes. Table 3 lists the frequency of use of the underlying concepts within each explanatory theme, ranked according to the most frequently used. This shows that the three most frequent underlying concepts used were those in the guidebook theme.

All nurses referred to the book in some way in

Table 1 Overall use of explanatory themes in answer texts

Theme

Number of nurses using this theme as a proportion of total number of nurses

The guidebook

The validity of the programme Models underpinning the programme

The nurses role in the programme
Table 2 Frequency of use of combinations of explanatory themes in answer texts

Combinations of themes

Number of nurses using this theme as proportion of total number of nurses

Four themes

Three themes

$16 / 23(70 \%)$

$2 \times$ validity missing

$2 \times$ nurse's role missing

Two themes

$1 \times$ validity and models

missing

$2 \times$ models and nurse's role

missing
Table 3 Rank order of concepts in answer texts

\begin{tabular}{|c|c|c|c|}
\hline Rank & Concepts & $\begin{array}{l}\text { Explanatory } \\
\text { theme }\end{array}$ & $\begin{array}{l}\text { Number of } \\
\text { nurses using } \\
\text { this subtheme } \\
\text { as a proportion } \\
\text { of total number } \\
\text { of nurses }\end{array}$ \\
\hline 1 & Purpose of book & Book & $22 / 23(96 \%)$ \\
\hline $2=$ & Definition of book & Book & $21 / 23(91 \%)$ \\
\hline $2=$ & Contents of book & Book & $21 / 23(91 \%)$ \\
\hline 4 & $\begin{array}{l}\text { Feelings, thoughts } \\
\text { and behaviour }\end{array}$ & Models & $17 / 23(78 \%)$ \\
\hline $5=$ & Nurse as collaborator & Nurse & $13 / 23(57 \%)$ \\
\hline $5=$ & Formal evidence base & Validity & $13 / 23(57 \%)$ \\
\hline $7=$ & $\begin{array}{l}\text { Informal evidence } \\
\text { base }\end{array}$ & Validity & $11 / 23(50 \%)$ \\
\hline $7=$ & Nurse as guide & Nurse & $11 / 23(50 \%)$ \\
\hline $9=$ & $\begin{array}{l}\text { Ease of use of the } \\
\text { guide }\end{array}$ & Book & $10 / 23(43 \%)$ \\
\hline $9=$ & Nurse as explicator & Nurse & $10 / 23(43 \%)$ \\
\hline 11 & The publisher & Validity & $9 / 23(39 \%)$ \\
\hline 12 & The authors & Validity & $8 / 23(35 \%)$ \\
\hline 13 & The $2+1$ model & Models & $7 / 23(30 \%)$ \\
\hline 14 & Remoralization & Models & $5 / 23(22 \%)$ \\
\hline 15 & The research context & Validity & $4 / 23(18 \%)$ \\
\hline 16 & The nurse as reviewer & Nurse & $2 / 23(9 \%)$ \\
\hline
\end{tabular}

their explanations. In addition, the other three explanatory themes were used by more than $80 \%$ of nurses. All nurses used more than one theme with $70 \%$ using all four to explain the self-help treatment. While concepts of purpose, definition and content within the book theme were the most frequently used, the most common concepts for the other three themes were the three systems
$23 / 23(100 \%)$

$20 / 23(87 \%)$

$20 / 23(87 \%)$

$19 / 23(83 \%)$ 
emotional model, the nurse as collaborator and the formal evidence base.

\section{Discussion}

The results of this study demonstrate that practice nurses working as non-mental health practitioners in primary care can articulate a sophisticated and complex rationale for mental health practice in primary care after only a short 3-day training programme. After receiving training, practice nurses can demonstrate that they understand a multifaceted yet practical health technology rationale for self-help mental health care. Their rationale not only includes reference to the technology itself which one would expect - but also discussion of a number of relevant psychological models, the principle of an evidence base and the complex role which the nurse has to adopt in order to deliver mental health care. All the nurses referred to the guidebook in their explanations and more than $85 \%$ used at least two other distinct thematic rationales. No nurse used less than two explanatory themes. As well as direct reference to aspects of the guidebook, almost three-quarters of the nurses specifically mentioned the three systems model of emotion at the heart of the self-help book.

The advantage of the methodological framework adopted for this study is that the results reflect the nurses' own understanding of the process of 'selling' mental health self-care to patients in primary care. We chose not to analyse the nurses' answers using a predetermined answer guideline framework. The results are, therefore, qualitatively valid in that they are the views of nurses with minimal mental health and psychology experience and who are actually going to deliver the mental health care to their patients. The fact that nurses integrate multiple psychological models and varied sources of evidence into their explanations of care rather than deliver mere descriptions of the book may lead to patients' own understanding being enhanced. Improved patient engagement and treatment concordance may also follow. This is of particular importance considering the self-motivation required for self-help interventions.

In terms of nurses' understanding, the guidebook is the focus, the role of the nurse is clear and there is no indication of 'therapizing' - i.e., nurses operating as counsellors using models favoured by some mental health nurses where the 'therapeutic use of self' is seen as the major agent of change (e.g., Barker, 1999). This result is counter to the expectations of some nurses who came on the course expecting to be trained in basic non-directive counselling skills. In contrast, training nurses to use a health technology model moves us away from traditional mental health training courses which have attempted to replicate some of the skills of experienced therapists in order to produce 'watered down' versions of specialist practitioners. It can be argued that the health technology approach offers a better skills and cultural fit for delivering primary mental health care. As a consequence we have utilized the data from this study in the design of a manual for practice nurses wishing to deliver primary mental health care.

\section{Limitations}

The data from this study were collected at the end of the last day of the PHASE training course. The number of nurses was small and the passage of time may lead to a reduction in understanding and hence complexity of explanation. We also have no measure of implementation by the nurses of their understanding and written explanations with actual patients. Although the educators were able to observe and be satisfied with nurses' performance in skills practice role plays, unlike other studies (e.g., Gask et al., 1988) we have no formal measure of this. However, the nature of the skill under consideration - understanding and explanation of a self-help guide that exists mainly in the cognitive domain - renders it well suited to written assessment. We were not seeking to assess other more complex psychotherapeutic skills such as interpretation, indeed, we actively discouraged this during the training. Finally, although this study has given us some process data, we do not as yet know how effective nurses can be in delivering the selfhelp guide to patients with common mental health problems in primary care. This is currently being tested in the quantitative analysis of data from our RCT.

\section{Conclusion}

This small study has demonstrated that it is possible to educate practice nurses in mental health self-help techniques. Using a health technology approach, the training programme has equipped nurses with a complex understanding of not only 
the technology but also the underlying psychological and organizational models, the importance of an evidence base and their role in the programme. With key elements of primary care nursing practice focusing on patient education and the use of health technology (Richards et al., 2000), this education programme may represent a more consistent fit with current professional nursing practice in primary care. Educating nurses in this way may enable them to deliver effective mental health care and be one route to addressing current policy concerns in the Mental Health NSF (Department of Health, 1999) about mental health delivery in primary care. We await the result of our RCT to see if these results are translated into real benefits for at least some of the $90 \%$ of patients whose mental health care needs are addressed entirely in primary care.

\section{References}

Balestrieri, M., Williams, P. and Wilkinson, G. 1988: Specialist mental health treatment in general practice: a meta-analysis. Psychological Medicine 18, 711-17.

Barker, P. 1999: Psychiatric nursing: philosophy and practice. London: Churchill Livingstone.

Barkham, M., Shapiro, D.A., Hardy, G.E. and Rees, A. 1999: Psychotherapy in two-plus-one sessions: outcomes of a randomised controlled trial of cognitive-behavioral and psychodynamic-interpersonal therapy for subsyndromal depression. Journal of Consulting and Clinical Psychology 67, 201-11.

Bower, P., Richards, D.A. and Lovell, K. 2001: The clinical and cost effectiveness of self-help treatments for anxiety and depressive disorders in primary care: a systematic review. British Journal of General Practice 51, 838-45.

Burnard, P. 1991: A method of analysing interview transcripts in qualitative research. Nurse Education Today 11; 461-66.

Department of Health 1999: A National Service framework for mental health. London: Stationery Office.

Donnan, P., Hutchinson, A., Paxton, R., Grant, B. and Firth, M. 1990: Self help materials for anxiety: a randomised controlled trial in general practice. British Journal of General Practice 40, 498-501.

Donoghue, J.M. and Tylee, A.T. 1996: The treatment of depression: prescribing patterns of antidepressants in primary care in the United Kingdom. British Journal of Psychiatry 168, 164-68.

Friedli, K. and King, M. 1996: Counselling in general practice a review. Primary Care Psychiatry 2, 205-16.

Gask, L., Goldberg, D., Lesser, A.L. and Millar, T. 1988: Improving the psychiatric skills of the general practice trainee: an evaluation of a group training course. Medical Education $22,132-38$.
Ghosh, A. and Marks, I. 1987: Self treatment of agoraphobia by exposure. Behaviour Therapy 18, 3-16.

Goldberg, D. and Bridges, K. 1987: Screening for psychiatric illness in general practice: the general practitioner versus the screening questionnaire. Journal of the Royal College of General Practitioners 37, 15-18.

Goldberg, D. and Huxley, P. 1992: Common mental disorders: a biosocial model. London: Routledge.

Gray, R., Parr, A.M., Plummer, S., Sandford, T., Ritter, S., Mundt-Leach, R., Goldberg, D. and Gournay, K. 1999: A national survey of practice nurse involvement in mental health interventions. Journal of Advanced Nursing 30, 901-906.

Holdsworth, N. and Paxton, R. 1999: Managing anxiety and depression. A self help guide. London; The Mental Health Foundation.

Holdsworth, N., Paxton, R., Seidel, S., Thomson, D. and Shrubb, S. 1996: Parallel evaluations of new guidance materials for anxiety and depression in primary care. Journal of Mental Health 5, 195-207.

Howard, K.I., Kopta, S.M., Krause, M.S. and Orlinsky, D.E. 1986: The dose-effect relationship in psychotherapy. American Psychologist 41, 159-64.

Kiely, B. and McPherson, I. 1986: Stress self-help packages in primary care: a controlled trial evaluation. Journal of the Royal College of General Practitioners 36, 307-309.

Kupshik, G. and Fisher, C. 1999: Assisted bibliotherapy: effective, efficient treatment for moderate anxiety problems. British Journal of General Practice 49, 47-48.

Marks, I.M. 1987: Fears, phobias and rituals. Oxford: Oxford University Press.

Mead, N., Bower, P. and Gask, L. 1997: Emotional problems in primary care: what is the potential for increasing the role of nurses? Journal of Advanced Nursing 26, 879-90.

Milne, D. and Covitz, F. 1988: A comparative evaluation of anxiety management materials in general practice. Health Education Journal 47, 67-69.

Mynors-Wallis, L.M., Gath, D.H., Day, A. and Baker, F. 2000: Randomised controlled trial of problem solving treatment, antidepressant medication, and combined treatment for major depression in primary care. British Medical Journal 320, 2630.

Nolan, P., Murray, E. and Dallender, J. 1999: Practice nurses' perceptions of services for clients with psychological problems in primary care. International Journal of Nursing Studies 36, 97-104.

Patel. A. and Knapp, M. 1998: Costs of mental illness in England. PSSRU Mental Health Review 5, 4-10.

Peveler, R., George, C., Kinmonth, A., Campbell., M. and Thompson, C. 1999: Effect of antidepressant drug counselling and information leaflets on adherence to drug treatment in primary care: randomised controlled trial. British Medical Journal, 319, 612-15.

PHASE Group 1999: A randomised controlled trial of the efficacy of patients receiving a brief self-help training package by practice nurses in primary care settings. (Study funded by the NHSExecutive N\&Y Region. In progress). 
Priest, R., Vize, C., Roberts, A., Roberts, M. and Tylee, A. 1996: Lay people's attitudes to treatment of depression: results of opinion poll for Defeat Depression Campaign just before its launch. British Medical Journal 313, 858-59.

Richards, A., Carley, J., Jenkins-Clarke, S. and Richards, D.A. 2000: Skill mix between nurses and doctors working in primary care-delegation or allocation: a review of the literature. International Journal of Nursing Studies 37, 185-97.

Roth, A. and Fonagy, P. 1996. What works for whom? A critical review of psychotherapy research. London: Guildford.

Sorby, N., Reavley, W. and Huber, J. 1991: Self help programme for anxiety in general practice: controlled trial of an anxiety management booklet. British Journal of General Practice 41, 417-20.

Thomas, R.V.R. and Corney, R.H. 1993: The role of the practice nurse in mental health. Journal of Mental Health 2, $65-72$.

Thompson, C., Kinmonth, A.L., Stevens, L., Peveler, R.C., Stevens, A., Ostler, K.J, Pickering, R.M., Baker, N.G., Henson, A., Preece, J., Cooper, D. and Campbell, M.J. 2000: Effects of a clinical practice guideline and practice-based education on detection and outcome of depression in primary care:
Hampshire Depression Project randomised controlled trial. The Lancet 355, 185-91.

Tylee, A. 1998: Education of primary care team: RCGP senior mental health education fellowship and Defeat Depression campaign. In Jenkins, R. and Ustun, T.B. editors, Preventing mental illness. Chichester: Wiley, 219-27.

White, J. 1995: Stresspac: a controlled trial of a self-help package for anxiety disorders. Behavioural and Cognitive Psychotherapy 23, 89-107.

Williams, C. J., Milton, J., Strickland, P., Ardagh-Walter, N., Knapp, J., Wilson, S., Trigwell, P., Feldman, E. and Sims, A.C.P. 1997: Impact of medical school teaching on preregistration house officers' confidence in assessing and managing common psychological morbidity: three centre study. British Medical Journal 315, 917-18.

World Health Organization 1999: The World Health Report. Geneva: World Health Organization.

Worrall, G., Angel, J., Chaulk, P., Clarke, C. and Robbins, M. 1999: Effectiveness of an educational strategy to improve family physicians' detection and management of depression: a randomized controlled trial. Canadian Medical Association Journal 161, 37-40. 